\title{
Kidney Volume Associations with Subclinical Renal and Cardiovascular Disease: The Diabetes Heart Study
}

\author{
Anita M. Saran ${ }^{a}$ Fang-Chi Hsu ${ }^{b}$ Kurt K. Lohman ${ }^{b} \quad$ J. Jeffrey Carr ${ }^{c, d}$ \\ Donald W. Bowden ${ }^{a, e, f \quad L y n n e ~ E . ~ W a g e n k n e c h t ~}{ }^{c}$ Barry I. Freedman ${ }^{a}$ \\ Departments of ${ }^{\mathrm{a}}$ Internal Medicine/Nephrology, ${ }^{\mathrm{b}}$ Biostatistical Sciences, ${ }^{\mathrm{C}}$ Epidemiology and Prevention, \\ ${ }^{\mathrm{d}}$ Radiology, and ${ }^{\mathrm{e} B i o c h e m i s t r y}$ and ${ }^{\mathrm{f}}$ Center for Human Genomics, Wake Forest University School of Medicine, \\ Winston-Salem, N.C., USA
}

\section{Key Words \\ Kidney volume $\cdot$ Glomerular filtration rate $\cdot$ Diabetes mellitus - Coronary atherosclerosis - Aorta atherosclerosis • Multidetector computed tomography, calcified plaque measurements}

\begin{abstract}
Background: The prognostic significance of total kidney volume (TKV) in subjects with type 2 diabetes mellitus (T2DM) is unknown. Methods: One hundred and seventy unrelated Caucasians with T2DM underwent multidetectorrow computed tomography of the neck, chest, and abdomen to measure calcified plaque in the coronary artery (CorCP), carotid artery (CarCP), and infrarenal aorta (AorCP). Spearman's rank correlation coefficients were used to assess associations between TKV and subclinical renal and cardiovascular disease. Partial correlation coefficients were computed to adjust for the potential confounding effects of age, sex, body mass index, glomerular filtration rate (GFR), diabetes duration, and hemoglobin $A_{1 c}$. Values are expressed as mean \pm SD (median in parentheses). Results: The study group (51\% female) had a mean age of $62.9 \pm 8.5$ (62.3) years, a T2DM duration of $11.5 \pm 6.8$ (10.0) years, a urinary albumin:creatinine ratio of $109.9 \pm 396(17.6) \mathrm{mg} / \mathrm{g}$, a GFR of
\end{abstract}

\begin{tabular}{ll}
\hline KARGER & $\odot$ 2007 S. Karger AG, Basel \\
$\begin{array}{l}\text { Fax }+41613061234 \\
\begin{array}{l}\text { E-Mail karger@karger.ch } \\
\text { www.karger.com }\end{array}\end{array}$ & $\begin{array}{l}\text { Accessible online at: } \\
\text { www.karger.com/ajn }\end{array}$
\end{tabular}

$63.8 \pm 12.8(63.2) \mathrm{ml} / \mathrm{min}$, a TKV of $272.4 \pm 69.7(261.9) \mathrm{cm}^{3}$, CorCP 2,170 \pm 3,394 (653), CarCP $374 \pm 673$ (104), AorCP $14,569 \pm 17,480(8,370)$, and a carotid artery intima-media thickness of $0.70 \pm 0.14(0.68) \mathrm{mm}$. Adjusting for age, sex, body mass index, diabetes duration, GFR, and hemoglobin $A_{1 c}$, the TKV was significantly associated with AorCP $(r=0.20$, $\mathrm{p}=0.016$ ), but not with CorCP, CarCP, or carotid artery intimamedia thickness (all $p \geq 0.25$ ). No significant associations were detected between TKV and blood pressure or albuminuria. Conclusions: In Caucasians with T2DM, TKV and calcified atherosclerotic plaque in the infrarenal abdominal aorta are positively associated. Common mechanisms linking renal matrix deposition with aortic atherosclerosis may underlie this association and require further study.

Copyright $\odot 2007$ S. Karger AG, Basel

\section{Introduction}

Traditionally, sonography has been the primary modality for the estimation of renal size; however, it is highly operator dependent [1]. It had been difficult to accurately measure kidney mass or volume until the development of noninvasive multidetector computerized tomography (MDCT). The prognostic value of total
Anita M. Saran, MD

Department of Internal Medicine/Nephrology

Wake Forest University School of Medicine, Medical Center Boulevard

Winston-Salem, NC 27157-1053 (USA)

Tel. +1 336716 7018, Fax +1 336716 4318, E-Mail asaran@wfubmc.edu 
kidney volume (TKV), measured using MDCT, and correlations between TKV and subclinical atherosclerosis in diabetes have not yet been evaluated.

Albuminuria and reduced glomerular filtration rate (GFR) are both potent and independent risk factors for cardiovascular disease (CVD). Diabetic subjects with albuminuria have been demonstrated to be far more likely to die from CVD than progress to elevated serum creatinine concentrations or renal replacement therapy [2]. Similarly, patients with chronic kidney disease (CKD) or end-stage renal disease (ESRD) are at high risk of suffering cardiac complications and stroke relative to subjects without nephropathy [3]. Calcified atherosclerotic plaque and carotid artery intima-media thickness (IMT) are widely accepted measures of subclinical atherosclerosis. Carotid artery IMT predicts incident coronary heart disease $[4,5]$, and the prevalence and incidence rates of coronary heart disease increase with increasing coronary artery calcified plaque (CorCP) [6,7]. To determine the usefulness of measuring TKV with MDCT, we determined correlations between TKV and albuminuria, blood pressure, and measures of subclinical atherosclerosis in unrelated Caucasians with type 2 diabetes mellitus (T2DM).

\section{Patients and Methods}

\section{Patient Population}

The Diabetes Heart Study (DHS) is a family-based investigation designed to identify genes producing susceptibility to subclinical CVD $[8,9]$. T2DM index cases (defined as diabetes onset $\geq 35$ years of age, in the absence of historical evidence of diabetic ketoacidosis) were recruited from hospitals and community clinics. One hundred and seventy unrelated Caucasian subjects with T2DM enrolled in the DHS with serum creatinine concentrations $\leq 1.6 \mathrm{mg} / \mathrm{dl}$ were randomly selected for measurement of TKV.

In the DHS, participants receive B mode ultrasound for carotid artery IMT, CT for measurement of calcified plaque in the coronary arteries, aortic arch, carotid arteries, and abdominal aorta and bone densitometry. The participants also undergo electrocardiography, fasting serum and urine chemistries, and interviews for past medical history, medications, and lifestyle.

\section{Imaging Methods}

CorCP, carotid artery calcified plaque (CarCP), and infrarenal aorta calcified plaque (AorCP) measurements were performed on a four-channel MDCT (MDCT4) with cardiac gating and capable of 500-ms temporal resolution using the segmented reconstruction algorithm (LightSpeed QXi; General Electric Medical Systems, Waukesha, Wisc., USA). The techniques for the coronary and carotid scans have previously been described in detail [8-10]. In brief, participants were placed in the supine position on the CT couch over a quality control calibration phantom (Image Analysis, Columbia, Ky., USA) for scans of the heart and abdomen. The participants were repositioned for the carotid artery bifurcation scan of the neck and placed in a head holder to aid in positioning.

The abdomen scan series was used to measure TKV. The technical factors for this series were: $120 \mathrm{kV}, 250 \mathrm{~mA}, 0.8$-second gantry rotation in helical mode $(7.5 \mathrm{~mm} / \mathrm{s}), 2.5-\mathrm{mm}$ slice thickness, and standard reconstruction kernel. The display field of view was $35 \mathrm{~cm}$, resulting in a pixel dimension of 0.68 by $0.68 \mathrm{~mm}$. The TKV was measured as the sum of the right and left kidneys determined separately, except in those with only one kidney and who denied prior surgical nephrectomy. The renal outlines were identified and measured by a single investigator (A.M.S.).

Computed tomography scans of all three vascular territories (coronary, carotid, and renal) were analyzed on an Advantage Windows Workstation with the SmartScores software package (General Electric Medical Systems) using a modified Agatston scoring method which adjusts for slice thickness and uses the conventional threshold of $130 \mathrm{HU}$.

Carotid artery IMT measurements were performed with the participants in the supine position using high-resolution B-mode carotid ultrasonography with a 7.5- $\mathrm{MHz}$ transducer and an AU5 ultrasound machine (Biosound Esaote, Indianapolis, Ind., USA), as previously described [9].

\section{Statistical Methods}

To demonstrate the demographic characteristics of the study population, the sample mean values and standard deviations were computed for the continuous characteristics [e.g., age, diabetes duration, body mass index (BMI), blood pressure, GFR, and serum cholesterol] and the measures of TKV and subclinical CVD (CorCP, CarCP, AorCP, and IMT). For the discrete demographic characteristics, such as gender and smoking, proportions were calculated.

This post hoc analysis was performed in a subgroup of unrelated Caucasian subjects participating in the DHS to assess for associations between TKV and measures of subclinical renal disease and CVD. Associations are presented by the Spearman rank correlation coefficient, since our major interest was to detect potential correlations between TKV and albuminuria, blood pressure, and measures of subclinical atherosclerosis. Spearman's rank correlation coefficient is a nonparametric measure of correlation. The TKV was not normally distributed in this cohort, thus assumptions about the distributions of the variables need not be made. Partial correlation coefficients were computed to adjust for the potential confounding effects of age, sex, BMI, diabetes duration, hemoglobin $\mathrm{A}_{1 \mathrm{c}}\left(\mathrm{HbA}_{\mathrm{lc}}\right)$, and GFR determined by the MDRD (Modification of Diet in Renal Disease) equation using demographic and serum variables: GFR $=170 \cdot$ (plasma creatinine $)^{-0.999} \cdot(\text { age })^{-0.176} \cdot(\text { blood urea nitrogen })^{-0.170} \cdot(\text { albumin })^{+0.318}$ - $(0.762$ if female $) \cdot(1.18$ if black). Partial correlation coefficients were obtained via computing the Spearman rank correlation coefficient between the residuals from models regressing TKV and measures of subclinical renal disease and CVD, respectively, onto age, sex, BMI, diabetes duration, $\mathrm{HbA}_{1 \mathrm{c}}$, and GFR. A simple scatter plot without covariate adjustment does not take into account the effect of the other covariates in the model. Thus, an untransformed added variable plot was used to demonstrate the association for the phenotype of interest (e.g., calcified plaque in a given vascular bed), after adjusting for other covariates. It is calculated as the residual from regressing TKV against all the covariates, omitting the single measure of subclinical renal disease or CVD. 

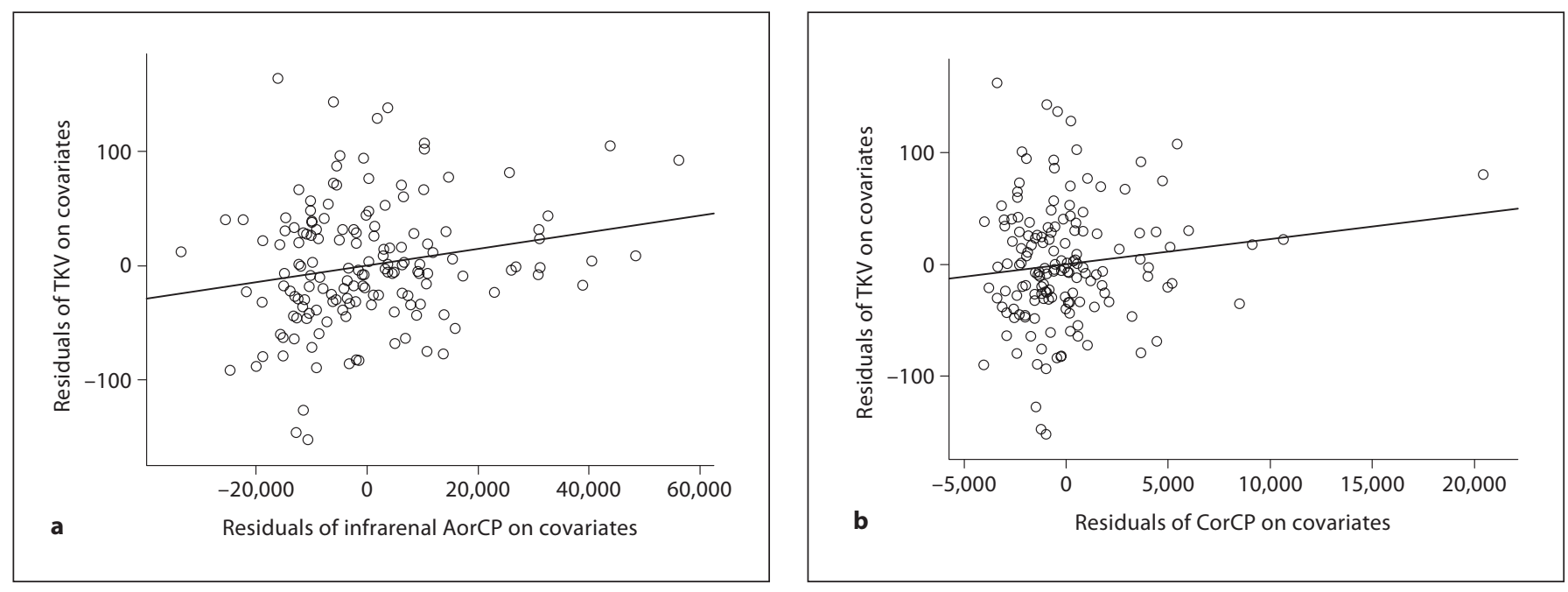

Fig. 1. a Association between TKV and infrarenal abdominal AorCP after adjustment for age, sex, diabetes duration, $\mathrm{HbA}_{1 \mathrm{c}}, \mathrm{BMI}$, and GFR. $\mathbf{b}$ Association between TKV and CorCP after adjustment for age, sex, diabetes duration, $\mathrm{HbA}_{1 \mathrm{c}}$, BMI, and GFR.

Next, the residual from regressing only the single measure of subclinical renal disease or CVD against the remaining covariates is determined. Finally, the residuals from the first step are plotted against the residuals from the second step. Regression lines are included in the figures to allow for improved visualization. Interpretation of these relationships is based on the Spearman rank correlation coefficient. SAS software version 9.1 (SAS Institute, Cary, N.C., USA) was used for all statistical analyses.

\section{Results}

Characteristics of the Caucasian diabetic study group included 51.2\% (87/170) females, $62.3 \%$ current or former smokers, $49.1 \%$ (82/170) were prescribed either angiotensin receptor blockers or angiotensin-converting enzyme inhibitors, and 49.4\% (84/170) received statins. Subjects had a mean age of $62.9 \pm 8.5$ (SD) (median 62.3) years, a duration of T2DM of $11.5 \pm 6.8$ (10.0) years, $\mathrm{HbA}_{1 \mathrm{c}} 7.5$ $\pm 1.4(7.3) \%$, BMI $32.2 \pm 6.3(31.7) \mathrm{kg} / \mathrm{m}^{2}$, modified MDRD equation GFR $63.8 \pm 12.8(63.2) \mathrm{ml} / \mathrm{min}$, and urinary albumin:creatinine ratio (ACR) $109.9 \pm 395.9$ (17.6) $\mathrm{mg} / \mathrm{g}$ (table 1). Radiographic characteristics included: TKV $272.4 \pm 69.7$ (SD) (median 261.9) $\mathrm{cm}^{3}$, CorCP $2,170 \pm 3,394$ (653), CarCP $374 \pm 673$ (104), AorCP $14,569 \pm 17,480(8,370)$, and carotid artery IMT $0.70 \pm$ $0.14(0.68) \mathrm{mm}$ (table 1). Two study participants had congenital solitary kidneys: 1 with TKV $185.3 \mathrm{~cm}^{3}$ and GFR $59.5 \mathrm{ml} / \mathrm{min}$ and 1 with TKV $156.2 \mathrm{~cm}^{3}$ and GFR 66.3 $\mathrm{ml} / \mathrm{min}$.
Crude associations between TKV and subclinical renal disease and CVD were computed after adjustment for age and sex (table 2). In this model, TKV was positively and marginally associated with AorCP $(\mathrm{r}=0.15 ; \mathrm{p}=$ 0.057 ), and a trend toward a positive association was observed for urinary ACR $(\mathrm{r}=0.14 ; \mathrm{p}=0.066)$ and CorCP $(\mathrm{r}=0.12 ; \mathrm{p}=0.117)$. No evidence of an association was observed between TKV and either CarCP or IMT (both $\mathrm{p}>0.6$ ).

Table 2 also contains the results of the fully adjusted model, accounting for the effects of age, sex, BMI, diabetes duration, $\mathrm{HbA}_{1 \mathrm{c}}$, and GFR. In the fully adjusted model, the association between TKV and AorCP increased $(\mathrm{r}=0.20, \mathrm{p}=0.016)$; however, the previously identified trends toward association between TKV and urinary $\mathrm{ACR}$ and CorCP were no longer evident (both $\mathrm{p}>0.2$ ), and no significant relationships were observed between TKV and CarCP, carotid artery IMT, and systolic or diastolic blood pressure.

Figure 1 reveals the distribution of residual effects from covariates plotted against residuals that remained from the effects of the amount of calcified plaque in the abdominal aorta (fig. 1a) and coronary artery (fig. 1b). The residuals of AorCP had a significant relationship with TKV after accounting for the effects of the other covariates $(\mathrm{p}=0.016)$, while residuals from CorCP did not $(\mathrm{p}>0.2)$. 
Table 1. Demographic characteristics of the study population ( $\mathrm{n}=170,87$ female and 83 male)

\begin{tabular}{lcc}
\hline Variable & Mean & SD \\
\hline Age, years & 62.9 & 8.5 \\
Diabetes duration, years & 11.5 & 6.8 \\
BMI, kg/m ${ }^{2}$ & 32.28 & 6.3 \\
Systolic blood pressure, mm Hg & 139.6 & 18.2 \\
Diastolic blood presssure, mm Hg & 70.9 & 9.9 \\
HbA $_{1 c} \%$ & 7.5 & 1.4 \\
Urinary ACR, mg/mg & 109.9 & 395.9 \\
GFR, ml/min & 63.8 & 12.8 \\
HDL cholesterol, mg/dl & 42.7 & 11.2 \\
LDL cholesterol, mg/dl & 103.3 & 34.3 \\
AorCP & $14,569.5$ & $17,480.1$ \\
CorCP & $2,169.6$ & $3,394.4$ \\
CarCP & 374.3 & 672.6 \\
Carotid artery IMT, mm & 0.7 & 0.14 \\
TKV, cm & & \\
\end{tabular}

\section{Discussion}

Computerized tomography scans of the chest and abdomen are noninvasive tests that permit precise quantification of TKV, as well as measurement of subclinical atherosclerosis in the coronary, aorta, and carotid artery circulations. Declining renal function and increasing albuminuria independently predict the amount of calcified atherosclerotic plaque [10] and an increased frequency of clinical CVD events [3]. Disagreement exists over how to best quantify renal function in individuals with CKD. These novel analyses explored potential TKV associations with subclinical CVD, blood pressure, and albuminuria.

We found the mean TKV to be $300.5 \pm 73.1 \mathrm{~cm}^{3}$ in males and $245.6 \pm 54.5 \mathrm{~cm}^{3}$ in females. Limited data exist regarding normal renal volumes measured by CT. In a study to determine the normal distribution of abdominal organ volume on 149 CT scans, mean organ volumes were $156.5 \mathrm{ml}$ for each kidney in females and $193.1 \mathrm{ml}$ for each kidney in males [11]. Therefore, these total volumes were larger than observed in our study; however, they were adjusted for patient height and weight. Unlike in the current report, 'thresholding' was not used to isolate the renal parenchyma and most of the CT scans were contrast enhanced. These factors may have contributed to the larger kidney sizes reported in that study. A recent study [12] used magnetic resonance imaging to assess $\mathrm{TKV}$ in patients without a history of kidney disease and

Kidney Volume in Diabetes Mellitus
Table 2. Spearman correlations ( $\mathrm{r}$ ) between $\mathrm{TKV}$ and covariates

\begin{tabular}{|c|c|c|c|c|}
\hline \multirow[t]{2}{*}{ Covariate } & \multicolumn{2}{|c|}{ Model 1} & \multicolumn{2}{|c|}{ Model 2} \\
\hline & $\mathrm{r}$ & $\mathrm{p}$ & $\mathrm{r}$ & $\mathrm{p}$ \\
\hline Urinary ACR & 0.14 & 0.07 & 0.06 & 0.45 \\
\hline CorCP & 0.12 & 0.12 & 0.10 & 0.25 \\
\hline CarCP & 0.03 & 0.66 & 0.08 & 0.31 \\
\hline AorCP & 0.15 & 0.06 & 0.20 & 0.02 \\
\hline Systolic blood pressure & 0.02 & 0.80 & -0.04 & 0.64 \\
\hline Diastolic blood pressure & -0.04 & 0.58 & -0.09 & 0.24 \\
\hline IMT & 0.035 & 0.66 & 0.07 & 0.42 \\
\hline Diabetes duration & 0.05 & 0.55 & 0.001 & 0.99 \\
\hline
\end{tabular}

Model 1: adjusted for age and sex; model 2: adjusted for age, sex, diabetes duration, $\mathrm{HbA}_{1 \mathrm{c}}$, BMI, and GFR.

found larger kidney volumes: an average kidney volume of 202 and $204 \mathrm{ml}$ for males and 154 and $156 \mathrm{ml}$ for females for the right and left kidneys, respectively.

The consistent positive association that was observed between TKV and infrarenal calcified atherosclerotic plaque may have implications for the coincident development of CKD and CVD in subjects with T2DM. The $\mathrm{TKV}$, reported as the sum of right and left kidney volumes, was not independently associated with urinary ACR, systolic or diastolic blood pressure, carotid artery IMT, or calcified plaque in the coronary or carotid circulations. Since all forms of CKD are associated with CVD, it would seem reasonable to expect negative associations to be present between TKV and calcified atherosclerotic plaque. Kidney size typically declines in most forms of chronic nephropathy, likely in concert with reduced renal mass and declining nephron number. Diabetic renal disease is unusual in that renal size typically increases early in the course of renal disease.

Increased kidney size has been noted to precede the development of proteinuria in type 1 diabetes. Nephromegaly is also a feature of T2DM with nephropathy. In a prospective cohort study of T2DM, kidney size was larger among diabetic versus matched nondiabetic participants and increased during the 6-year follow-up period [13]. In our study population, 111 of the 170 subjects had an ACR between 0 and $30 \mathrm{mg} / \mathrm{g}$, and only 7 had overt proteinuria (ACR $>300 \mathrm{mg} / \mathrm{g}$ ). Therefore, the DHS population with minimal proteinuria and relatively preserved renal function should allow for detection of relationships between TKV (as a marker of early diabetic nephropathy) and subclinical CVD. 
Aortic calcification is an important risk factor for coronary heart disease. In an autopsy study, Takasu et al. [14] demonstrated that aorta calcification on a nonenhanced CT was correlated with aortic intimal thickness and atheromatous degeneration. In 133 newly diagnosed individuals with T2DM, Siitonen et al. [15] found that diabetic males had more abdominal aortic calcification than nondiabetic males, and positive associations were observed between aorta calcium and ischemic changes on electrocardiogram. Aortic calcium is an independent predictor of incident coronary heart disease [16], and a 9-year prospective study [17] found that the prevalence of aorta calcium increased with age and was associated with a sixfold increase in risk of CVD events among men aged 45 years. Reaven et al. [18] detected an increase in the prevalence of peripheral arterial disease, coronary artery disease, and cerebrovascular disease in subjects with T2DM who had detectable abdominal aorta calcium. In hemodialysis patients, abdominal aortic calcification, as determined by lateral X-ray, was associated with increased cardiovascular and all-cause mortality [19]. Correlations between abdominal aorta calcification on standard flat-plate X-ray and electron beam CT coronary artery calcium have also been described [20].

Despite the association of TKV with AorCP, there was a lack of a relationship between TKV and CorCP, CarCP, or carotid artery IMT. Other reports revealed that abdominal aortic atherosclerosis is more strongly correlated with peripheral vascular disease $(p<0.0001)$ than with coronary disease $(\mathrm{p}<0.05)$, and correlations between aortic atherosclerosis and cerebrovascular disease were absent [14]. Autopsy studies also revealed that aortic atherosclerotic lesions likely precede development of cor- onary lesions [21] and that age is the strongest predictor for abdominal aortic calcification. Although the factors that are responsible for atherosclerosis in the abdominal aorta are clearly similar to those in the coronary and carotid circulations, the correlations between amounts of calcified plaque in these three vascular beds ranged from only 0.59 to 0.72 among all 1,125 diabetic participants in the DHS [22]. Several conventional CVD risk factors appear to have differential impacts on the development of calcified plaque in these three beds, particularly smoking, male gender, and history of prior myocardial infarction.

This study demonstrated strong positive associations between TKV and calcified atherosclerotic plaque in the infrarenal aorta, an association that was independent of kidney function. Additional studies will be necessary to determine whether common factors (e.g., oxidized lipid species) provide a common mechanism for development of abdominal AorCP and increased renal volume. Prospective studies are also needed to determine whether increased renal volumes as measured by abdominal CT are clinically predictive of future cardiovascular events in subjects with T2DM.

\section{Acknowledgments}

This study was supported in part by the General Clinical Research Center of the Wake Forest University School of Medicine (Grant MO1 RR07122) and by NIH Grants RO1 HL67348 (to D.W.B.) and RO1 AR48797 (to J.J.C.). The authors wish to thank our dedicated CT technologists at the Wake Forest University Baptist Medical Center.

\section{References}

1 O’Neill W, Baumgarten A: Imaging. Am J Kidney Dis 2003;42:601-604.

-2 Adler AI, Stevens RJ, Manley SE, Bilous RW, Cull CA, Holman RR; UKPDS Group: Development and progression of nephropathy in type 2 diabetes: the United Kingdom Prospective Diabetes Study (UKPDS 64). Kidney Int 2003;63:225-232.

- 3 Go AS, Chertow GM, Fan D, McCulloch CE, Hsu CY: Chronic kidney disease and the risks of death, cardiovascular events, and hospitalization. N Engl J Med 2004;351: 1296-1305.
4 Chambless LE, Heiss G, Folsom AR, Rosamond WD, Szklo M, Sharrett AR, Clegg LX: An association of coronary heart disease incidence with carotid arterial wall thickness and major risk factors: The Atherosclerosis Risk in Communities (ARIC) Study, 19871993. Am J Epidemiol 1997;146:483-494.

5 O'Leary DH, Polak JF, Kronmal RA, Mano lio TA, Burke GL, Wolfson SK: Carotid-artery intima and media thickness as a risk factor for myocardial infarction and stroke in older adults. N Engl J Med 1999;340:14-22.
-6 Raggi P, Callister TQ, Cooil B, He ZX, Lippolis NJ, Russo DJ, Zelinger A, Mahmarian JJ: Identification of patients at increased risk of first unheralded acute myocardial infarction by electron-beam computed tomography. Circulation 2000;101:850-855.

7 Arad Y, Spadaro LA, Goodman K, Newstein D, Guerci AD: Prediction of coronary events with electron beam computed tomography. J Am Coll Cardiol 2000;36:1253-1260.

$\checkmark 8$ Wagenknecht L, Bowden D, Carr J, Langefeld C, Freedman B, Rich S: Familial aggregation of coronary artery calcium in families with type 2 diabetes. Diabetes 2001;50:861866. 
-9 Lange LA, Bowden DW, Langefeld CD, Wagenknecht LE, Carr JJ, Rich SS, Riley WA, Freedman BI: Heritability of carotid artery intima-medial thickness in type 2 diabetes. Stroke 2002;33:1876-1881.

- 10 Freedman BI, Langefeld CD, Lohman KK, Bowden DW, Carr JJ, Rich SS, Wagenknecht LE: Relationship between albuminuria and cardiovascular disease in type 2 diabetes. J Am Soc Nephol 2005;16:2156-2161.

11 Geraghty EM, Boone JM, McGahan JP, Jain $\mathrm{K}$ : Normal organ volume assessment from abdominal CT. Abdom Imaging 2004;29: 482-490.

12 Cheong B, Muthupillai R, Rubin MF, Flamm SD: Normal values for renal length and volume as measured by magnetic resonance imaging. Clin J Am Soc Nephrol 2007;2:3845.

13 Wirta O, Pasternack A, Laippala P, Turjanamaa V: Glomerular filtration rate and kidney size after six years disease duration in non-insulin-dependent diabetic subjects. Clin Nephrol 1996;45:10-17.
4 Takasu J, Takanashi K, Naito S, Onishi M, Miyazaki A, Aoyagi Y, Morooka N, Masuda Y, Inagaki Y: Evaluation of morphological changes of the atherosclerotic aorta by enhanced computed tomography. Atherosclerosis 1992;97:107-121.

15 Siitonen O, Uusitupa M, Pyorala K, Lansimies E, Voutilainen E: Aortic calcifications and their relationship to coronary heart disease and cardiovascular risk factors in patients with newly diagnosed non-insulin-dependent diabetes and nondiabetic subjects. Cardiology 1987;74:335-343.

16 Wilson PW, Kauppila LI, O’Donnell CJ, Kiel DP, Hannan M, Polak JM, Cupples LA: Abdominal aortic calcific deposits are an important predictor of vascular morbidity and mortality. Circulation 2001;103:1529-1534

17 Witteman JC, Kok FJ, van Saase JL, Valkenburg HA: Aortic calcification as a predictor of cardiovascular mortality. Lancet 1986;2: 1120-1122.

18 Reaven PD, Sacks J; Investigators for the VADT: Coronary artery and abdominal aortic calcifications are associated with cardiovascular disease in type 2 diabetes. Diabetologia 2005;48:379-385.
9 Okuno S, Ishimura E, Kitatani K, Fujino Y, Kohno K, Maeno Y, Maekawa K, Yamakawa T, Imanishi Y, Inaba M, Nishizawa Y: Presence of abdominal aortic calcification is significantly associated with all-cause and cardiovascular mortality in maintenance hemodialysis patients. Am J Kidney Dis 2007;49:417-425.

20 Bellasi A, Ferramosca E, Muntner P, Ratti C, Wildman RP, Block GA, Raggi P: Correlation of simple imaging tests and coronary artery calcium measured by computed tomography in hemodialysis patients. Kidney Int 2006;70:1623-1628.

21 PDAY Research Group: Natural history of aortic and coronary atherosclerotic lesions in youth. Arterioscler Thromb 1993;13: 1291-1298.

22 Bowden DW, Rudock M, Ziegler J, Lehtinen $\mathrm{AB}, \mathrm{Xu}$ J, Wagenknecht LE, Herrington D, Rich SS, Freedman BI, Carr JJ, Langefeld CD: Coincident linkage of type 2 diabetes, metabolic syndrome, and measures of cardiovascular disease in a genome scan of the Diabetes Heart Study. Diabetes 2006;55:19851994. 